\title{
Fabrication of Ag and Au nanoparticles in cross-linked polymer microgels for their comparative catalytic study
}

\author{
Luqman Ali Shah ${ }^{1, *}$, Murtaza SaYed ${ }^{1}$, Mohammad SiddiQ $^{2}$ \\ ${ }^{1}$ National Centre of Excellence in Physical Chemistry, University of Peshawar, Peshawar 25120, Pakistan \\ ${ }^{2}$ Department of Chemistry, Quaid-i-Azam University, Islamabad 45320, Pakistan
}

\begin{abstract}
Three dimensional cross-linked polymer microgels with temperature responsive N-isopropyl acrylamide (NIPAM) and $\mathrm{pH}$ sensitive methacrylic acid (MAA) were successfully synthesized by free radical emulsion polymerization with different amounts of MAA. Silver and gold nanoparticles with the size of $6.5 \mathrm{~nm}$ and $3.5 \mathrm{~nm}( \pm 0.5 \mathrm{~nm})$, respectively were homogeneously reduced inside these materials by chemical reduction method at $\mathrm{pH} 2.78$ and 8.36 for the preparation of hybrid materials. The samples were characterized by FT-IR, DLS and TEM techniques. The catalytic activity of the hybrid materials was investigated for the reduction of 4-nitrophenol (4-NP) using $\mathrm{NaBH}_{4}$ as reducing agent by UV-Vis spectroscopy. The hybrid polymer network synthesized at $\mathrm{pH} 8.36$ showed enhanced catalytic efficiency compared to the catalysts synthesized at $\mathrm{pH} 2.78$. In this study, it has been stated that the catalyst activity strongly depends on the amount of MAA, pH value during synthesis and the type of entrapped metal nanoparticles.
\end{abstract}

Keywords: cross-linked polymer microgels; free radical polymerization; metal nanoparticles; catalytic activity; comparative study

\section{Introduction}

Due to their intelligent and smart behavior, cross-linked polymer microgels received a significant interest in many fields of applications e.g. drug delivery [1], wound dressing [2], sensors [3], artificial mussels [4], optical devices [5], catalysis [6], lithium batteries [7], nanoreactors [8], etc. Polymer microgels are 3D cross-linked network showing reversible responsive behavior with the environmental stimuli such as temperature $[9,10]$, $\mathrm{pH}$ [11], ionic strength [12], enzymes [5], glucose [13], electric current [14], etc. In catalysis, microgel particles are used as a shelter for the fabrication of metal nanoparticles (NPs). As metal NPs received notable attention due to their catalytic performance, their small size and large surface, because of high surface energy the particles, are unstable and undergo aggregation which restricts their catalytic properties. So, to avoid the aggregation process, cross-linked polymer microgel networks have been designed. These microgel

*E-mail: luqman_alisha@yahoo.com particles not only provide a support for metal NPs, but also change their properties by changing their own structure with the application of external stimuli. The entrapment of metal particles inside the microgel network enables us to produce hybrid systems which can be used anywhere in a controlled fashion for better yield.

The hybrid systems with responsive tunable properties can be used as catalysts for different reactions. The combination of two or more monomers in a polymer chain gives multiresponsive character to the polymer network, which enhances their use for multiple purposes.

Currently, Wu et al. [15] immobilized copper NPs on responsive chitosan based microgels and applied them as a catalyst for a model azide-alkyne $[3+2]$-cycloaddition reaction. They found that the catalyst efficiency can be tuned to some extent with changing swelling and de-swelling transitions of polymer gels. Liu et al. [16] investigated the temperature responsive catalytic activity of Ag NPs loaded in temperature sensitive poly(N-isopropyl acrylamide) microgels. They 
Table 1. Feed ratios, particle size and zeta potentials of the synthesized copolymer microgels at $25^{\circ} \mathrm{C}$.

\begin{tabular}{ccccccc}
\hline Sample code & $\begin{array}{c}\text { Feed ratio of } \\
\text { NIPAM:MAA:BIS }\end{array}$ & $\begin{array}{c}\text { Total } \\
\text { moles }\end{array}$ & $\mathrm{pH}$ & $\begin{array}{c}\text { Zeta } \\
\text { potential [mV] }\end{array}$ & $\begin{array}{c}\text { Mean diameter } \\
\text { by DLS [nm] }\end{array}$ & $\begin{array}{c}\text { Mean size } \\
\text { by TEM [nm] }\end{array}$ \\
\hline \hline p(NIPAM-co-MAA)-1 & \multirow{2}{*}{$85: 10: 5$} & 0.011 & 2.78 & -3.13 & 148 & 108 \\
& & & 8.36 & -22.3 & 293 & 262 \\
p(NIPAM-co-MAA)-2 & \multirow{2}{*}{$80: 15: 5$} & 0.011 & 2.78 & -4.09 & 170 & 123 \\
& & & 8.36 & -23.9 & 371 & 348 \\
\hline
\end{tabular}

showed that the catalytic activity of embedded Ag NPs can be modulated at four stages by variation of temperature from $20{ }^{\circ} \mathrm{C}$ to $45{ }^{\circ} \mathrm{C}$, but more efficiently above $35{ }^{\circ} \mathrm{C}$. Contin et al. [17] used laponite nanoclay as a cationic exchanger for metal NPs ( $\mathrm{Pd}, \mathrm{Pt}$ and $\mathrm{Au}$ ) which upon reduction yielded ternary nanocomposites composed both of metal particles and nanoclays. The materials were applied as catalysts for standard cross coupling reactions in aqueous medium. Gema et al. [18] prepared PNIPAM/gold hybrid hydrogels by using catechol redox chemistry. The catalytic activity of hybrid materials was studied by using reduction of 4-nitrophenol (4-NP). The results revealed high catalyst efficiency at $25{ }^{\circ} \mathrm{C}$ compared to $38{ }^{\circ} \mathrm{C}$ due to the shrinkage of PNIPAM structure. Sahiner [19] fabricated Co and Ni NPs inside cationic poly (ethylene imine) microgel particles prepared by microemulsion polymerization. The synthesized species were used as catalysis medium for reduction of 4-NP to 4-aminophenol. Cationic copolymer microgels composed of vinyl imidazole and $\mathrm{N}$-isopropylacrylamide moieties were prepared by us [20] and successfully applied for the fabrication of Ag NPs. The materials showed good catalytic activity with high stability in the entire $\mathrm{pH}$ range. Recently, in another study [21], Ag and $\mathrm{Au}$ nanoparticles were reduced inside ter-copolymer microgels at $10{ }^{\circ} \mathrm{C}$ and $40{ }^{\circ} \mathrm{C}$ and their catalytic activities were studied in detail for the degradation of 4-NP, methylene blue and congo red. The catalysts synthesized at $40{ }^{\circ} \mathrm{C}$ showed enhanced catalytic activity due to their direct exposure to reactant molecules.

In this work, for the first time, we fabricated $\mathrm{Ag}$ and $\mathrm{Au}$ NPs at $\mathrm{pH} 2.78$ and 8.36 in copolymer poly(N-isopropylacrylamide-co-methacrylic acid) P(NIPAM-co-MAA) microgels. The effect of MAA amount, temperature and $\mathrm{pH}$ sensitive behavior on the particle size has been investigated in detail and results obtained have been compared with the literature. The hybrid microgels were tested for their comparative catalytic activity towards the reduction of 4-NP as a model reaction.

\section{Experimental}

\subsection{Chemicals}

$\mathrm{N}$-isopropylacrylamide (NIPAM, $98 \%$, TCI) was recrystallized from a mixture of toluene and $n$-hexane $(\mathrm{V}: \mathrm{V}=1: 3)$. Methacrylic acid (MAA, $98 \%$, TCI) was passed through a neutral alumina column to remove inhibitors. $\mathrm{N}, \mathrm{N}$-methylene bisacrylamide (BIS, ACROS), silver nitrate $\left(\mathrm{AgNO}_{3}\right)$ and chloroauric acid $\left(\mathrm{HAuCl}_{4} \cdot \mathrm{H}_{2} \mathrm{O}\right)$ were purchased from ALDRICH. Ammonium peroxodisulfate (APS), sodium dodecyl sulfate (SDS) and 4-nitrophenol (4-NP) were all purchased from TCI and were used as received. Double distilled water (DDW) was used during all the experiments.

\subsection{Synthesis of p(NIPAM-co-MAA) copolymer microgels}

The synthesis of $\mathrm{p}(\mathrm{NIPAM}-\mathrm{co}-\mathrm{MAA})$ copolymer microgels was carried out by free radical emulsion polymerization reported in the literature [22] with slight modification. Typically, the required amount of monomers NIPAM and MAA (listed in Table 1), $84.7 \mathrm{mg}$ of BIS and $57 \mathrm{mg}$ of SDS were dissolved in $100 \mathrm{~mL}$ of deionized water and stirred for $1 \mathrm{~h}$. The mixture was purged with nitrogen, heated to $65{ }^{\circ} \mathrm{C}$, and $5 \mathrm{~mL}$ aqueous solution of APS $(65 \mathrm{mg} / 5 \mathrm{~mL})$ was added for initiation. After complete polymerization, the mixture was purified by decantation, centrifugation and transferred 
to a dialysis bag with a molecular weight cutoff (MWCO) of 14000 for dialysis. The bag was immersed into distilled water for 10 days at constant stirring; water was continuously replaced twice a day. The resultant $\mathrm{p}$ (NIPAM-co-MAA) copolymers were lyophilized and collected for further use.

\subsection{Synthesis of metal entrapped p(NIPAM-co-MAA)-Ag and p(NIPAM- co-MAA)-Au hybrid microgels}

Polymer hybrid microgels p(NIPAM-coMAA)-Ag and p(NIPAM-co-MAA)-Au, were synthesized at $\mathrm{pH} 2.78$ and 8.36 from pure microgels. $2 \mathrm{mg}$ of synthesized pure copolymer microgels were diluted by adding $35 \mathrm{~mL}$ of pure deionized water in two three-necked flasks. After stirring for $3 \mathrm{~h}$, the $\mathrm{pH}$ of both solutions was adjusted to 2.78 by adding very dilute $\mathrm{HCl}$ solution. Then, one solution was charged with $5 \mathrm{~mL}$ of chloroauric acid $\left(\mathrm{HAuCl}_{4} \cdot \mathrm{H}_{2} \mathrm{O}, 1 \mathrm{mM}\right)$ to one flask and the other one with $5 \mathrm{~mL}$ of silver nitrate $\left(\mathrm{AgNO}_{3}, 1 \mathrm{mM}\right)$ to another flask. The stirring was continued for another $1 \mathrm{~h}$ with continuous purging of $\mathrm{N}_{2}$ for 0.75 h. $4 \mathrm{~mL}$ freshly prepared $\mathrm{NaBH}_{4}$ solution $\left(0.13 \mathrm{~g} / 8 \mathrm{~mL}\right.$ of $\left.\mathrm{H}_{2} \mathrm{O}\right)$ was added to each flask. The color of the solutions was changed rapidly. The reactions were conducted for $3 \mathrm{~h}$ to completion; the samples were purified by dialysis for $2 \mathrm{~h}$, dried under vacuum and stored for characterization. The same procedure was followed for hybrid microgels synthesized at $\mathrm{pH} 8.36$ using very dilute solution of sodium hydroxide for $\mathrm{pH}$ adjustment.

\subsection{Catalytic activity of metal entrapped hybrid microgels}

To evaluate the catalytic activity of p(NIPAMco-MAA)-Ag and p(NIPAM-co-MAA)-Au, $0.06 \mathrm{mmol}(8.22 \mathrm{mg})$ of 4-NP was dissolved in $100 \mathrm{~mL}$ of deionized water and stirred to get a clear light yellow colored solution. $5.3 \mathrm{mmol}(0.20 \mathrm{~g})$ of $\mathrm{NaBH}_{4}$ was added to this solution. $2.5 \mathrm{~mL}$ of the synthesized solution was transferred into a quartz cell with a path length of $1 \mathrm{~cm}$, followed by adding $0.1 \mathrm{~mL}$ of $0.2 \mathrm{mM}$ p(NIPAM-coMAA)-Ag and p(NIPAM-co-MAA)-Au aqueous solution separately for each reduction process. UV-Vis spectrophotometer was used to monitor the reaction. The reaction rate constant $k_{a p p}$ could be calculated from the relationship between the absorption at $400 \mathrm{~nm}$ and the reaction time.

\subsection{Characterization}

The FT-IR spectra were recorded on a NEXUS-470 spectrometer to identify different functional groups present in the microgels. The samples were vacuum dried on silicon chip before analysis. The hydrodynamic diameter and Zeta potentials were measured on a Malvern autosizer 4700 instrument; the samples were filtered through hydrophilic Millipore Millex-HV filters with a pore size of $0.45 \mu \mathrm{m}$ to remove any dust particles. Transmission electron microscopy (TEM) images were obtained on a JEOL JEM $2100 \mathrm{~F}$ transmission electron microscope, and samples for TEM measurements were made by casting one drop of the sample solution on a carbon copper grid. The ultraviolet visible absorption analysis was carried out using a PerkinElmer Lambda 750 UV-Vis-NIR spectrometer.

\section{Results and discussion}

\subsection{Synthesis of microgels}

The successful copolymerization and microgel formation of NIPAM and MAA were confirmed by FT-IR spectroscopy. The FT-IR spectra for composition 1 (both pure and hybrid) are shown in Fig. 1, the FT-IR spectrum of p(NIPAMco-MAA) shows a broad $\mathrm{N}-\mathrm{H}$ stretching vibration peak at $3300 \mathrm{~cm}^{-1}$, a clear $\mathrm{C}-\mathrm{N}$ stretching vibration peak at $1459 \mathrm{~cm}^{-1}$ and an obvious $\mathrm{C}=\mathrm{O}$ (amide) stretching vibration peak at $1635 \mathrm{~cm}^{-1}$, however, no characteristic peaks of vinyl functionality indicates the complete disappearance of initial monomers. After combination with $\mathrm{Ag}$ and $\mathrm{Au}$ nanoparticles, the $\mathrm{C}=\mathrm{O}$ stretching vibration peak of $p$ (NIPAM-co-MAA)-Ag and $p$ (NIPAMco-MAA)-Au was shifted from $1651 \mathrm{~cm}^{-1}$ to $1642 \mathrm{~cm}^{-1}$ and $1635 \mathrm{~cm}^{-1}$, respectively, indicating that the $\mathrm{Ag}$ and $\mathrm{Au}$ nanoparticles were successfully integrated with $\mathrm{C}=\mathrm{O}$ groups, while all other 
peaks of corresponding functional groups appeared at the same position. The entrapment, existence and size of metal particles was also confirmed by transmission electron microscopy.

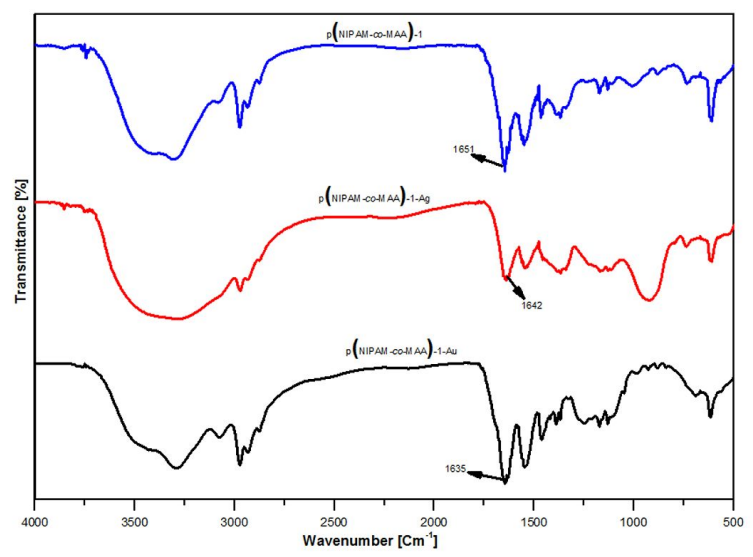

Fig. 1. FT-IR spectra of pure and metal entrapped hybrid microgels.

\subsection{Sensitive behavior of microgels}

The presence of $\mathrm{pH}$-responsive MAA and temperature sensitive NIPAM, introduce a remarkable $\mathrm{pH}$ and temperature sensitive behavior in p(NIPAM-co-MAA)s polymer microgels, which changes their size in different environmental conditions. It could be seen from Fig. 2 that the size of $\mathrm{p}$ (NIPAM-co-MAA)s determined by DLS was increased with the increase in $\mathrm{pH}$ of the microgel solution. This was caused by the deprotonation of carboxyl group in the basic media, which induced electrostatic repulsive interactions and caused extension of the polymer chains and size increase [23]. The critical volume phase transition $\mathrm{pH}$ of $\mathrm{p}$ (NIPAM-co-MAA)s was approximately 4.5; below this value the particles were small in size of about $148 \mathrm{~nm}$ and $170 \mathrm{~nm}$ for p(NIPAM-co-MAA)-1 and p(NIPAM-co-MAA)-2, respectively. At $\mathrm{pH} 2.78$, above the transition $\mathrm{pH}$, the particles reached the maximum size value shown in Fig. 2.

Furthermore, with the increase of MAA content in $\mathrm{p}$ (NIPAM-co-MAA)s, the $\mathrm{pH}$-dependent size change was more distinct due to the increase in surface charge on the particles, which was further
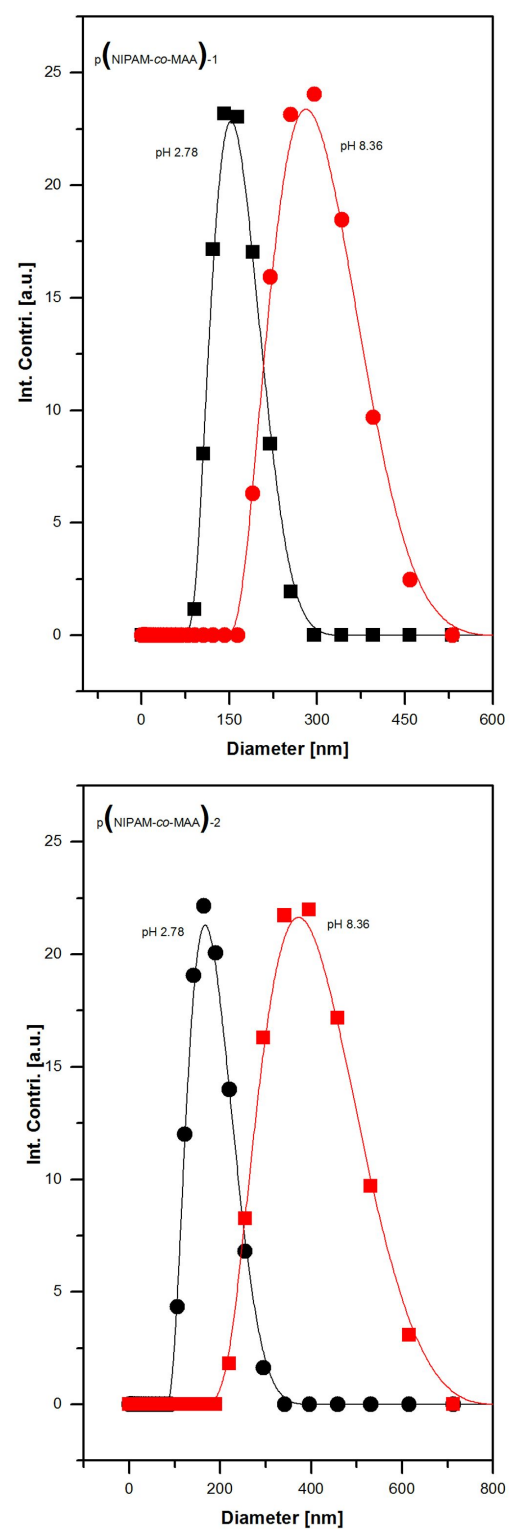

Fig. 2. Particle size of $\mathrm{p}$ (NIPAM-co-MAA)s copolymer microgels at different $\mathrm{pH}$ values.

confirmed by measuring zeta potentials at $\mathrm{pH} 2.78$ and 8.36. The particles size was also confirmed by TEM analysis (Fig. 3). The size obtained by TEM was less than that of DLS, and this was due to the dry nature of the samples considered for TEM analysis whereas DLS calculated hydrodynamic size of particles in solution. The zeta potentials and particle size values obtained at room temperature for $\mathrm{p}$ (NIPAM-co-MAA)-1 and $\mathrm{p}$ (NIPAMco-MAA)-2 are tabulated in Table 1. 

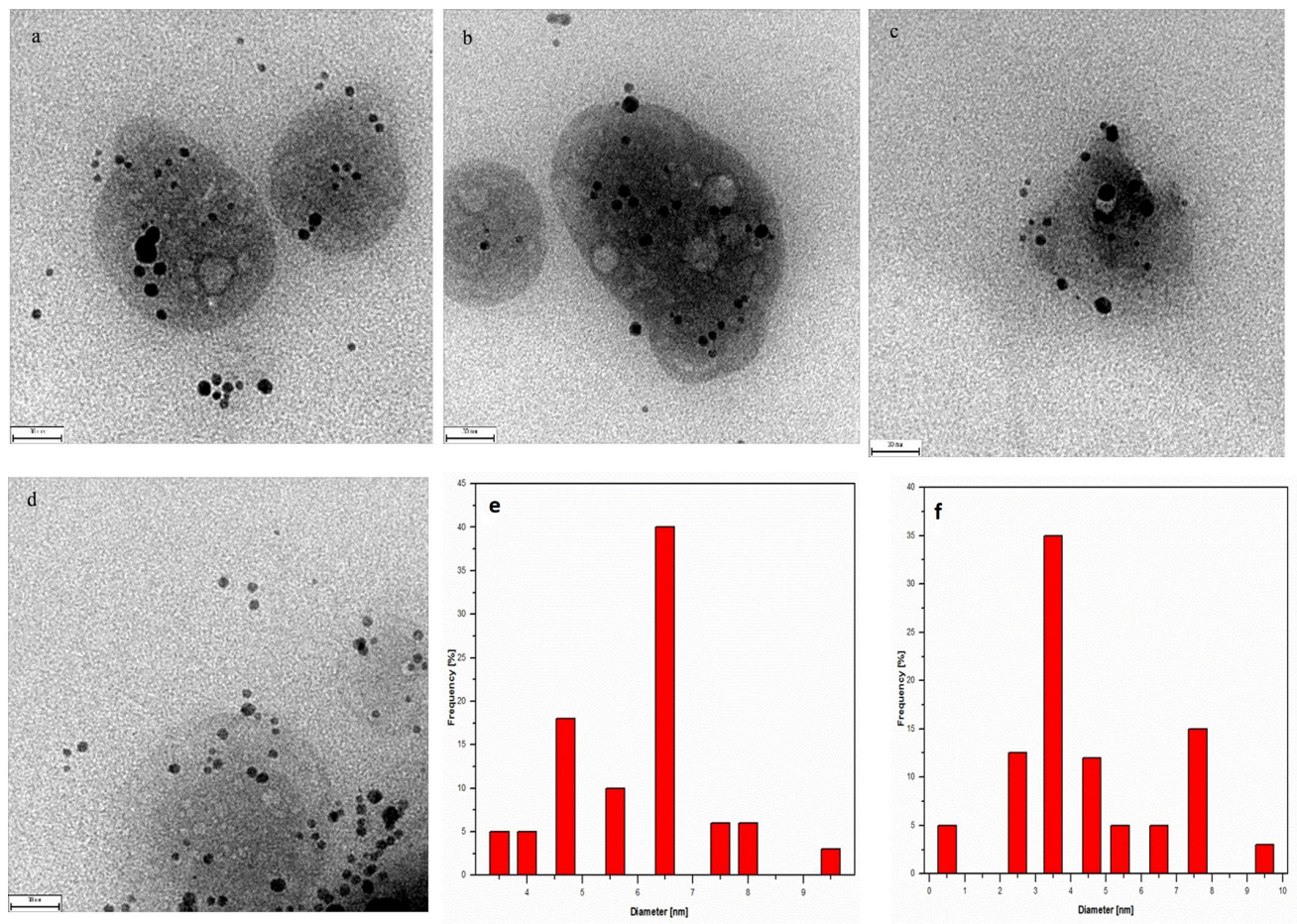

Fig. 3. TEM images of metal entrapped microgel particles at $\mathrm{pH} 2.78$ with their size distribution (a) p(NIPAMco-MAA)-1-Ag (b) p(NIPAM-co-MAA)-2-Ag (c) p(PNIPAM-co-MAA)-1-Au (d) p(NIPAM-co-MAA)-2$\mathrm{Au}$ (e) size distribution of Ag nanoparticles in p(NIPAM-co-MAA)-1-Ag and (f) size distribution of $\mathrm{Au}$ nanoparticles in p(NIPAM-co-MAA)-1-Au.

The thermal sensitivity of $\mathrm{p}$ (NIPAM-co-MAA)s was investigated at $\mathrm{pH} 2.78$ and 8.36. At $\mathrm{pH}$ 2.78 , both $\mathrm{p}$ (NIPAM-co-MAA)s showed obviously pronounced thermal responsibility (Fig. $4 \mathrm{a}$ and Fig. 4c), due to the weak electrostatic interactions of MAA segment. A clear volume phase transition temperature (VPTT) was observed which decreased at the high MAA content in the copolymer composition. The VPTT for pure NIPAM is $32{ }^{\circ} \mathrm{C}$, the introduction of $15 \%$ MAA, lowered VPTT to $29{ }^{\circ} \mathrm{C}$. The value of VPTT is determined by the balance between the hydrophilic and hydrophobic interaction of copolymers. Thus, the increase of hydrophobic MAA monomer leads to a shift of VPTT to a lower temperature. No aggregation was observed for both compositions at high temperature up to $50{ }^{\circ} \mathrm{C}$, which was confirmed by the presence of some charge on the surface of particles at low $\mathrm{pH}$ calculated from zeta potential values shown in Table 1. However, aggregation of particles at low $\mathrm{pH}$ and high temperature was reported for acrylic acid (AA) in a previous study [22]. At pH 8.36, the strong electrostatic repulsive forces shifted the VPTT to a higher values and only a slight decrease in particle size was observed at $50{ }^{\circ} \mathrm{C}$ (Fig. $4 \mathrm{~b}$ and Fig. 4d). This decrease is due to the expelling of water molecules from polymer network. The VPTT for $\mathrm{p}$ (NIPAM-co-MAA)-2 was found again less than for $\mathrm{p}$ (NIPAM-co-MAA)-1 at $\mathrm{pH} 8.36$ due to the high amount of MAA.

\subsection{Discussion on catalytic tests}

The particle size which is a function of charge on the particle surface is responsible for the entrapment of metal nanoparticles inside the polymer network. It was confirmed by synthesizing hybrid microgels at pH 2.78 and 8.36, and studying their comparative catalytic activity. The catalytic activity was examined for all prepared hybrid microgels used as catalysts for the reduction of 4-NP to 4-aminophenol (4-AP) in aqueous solution 

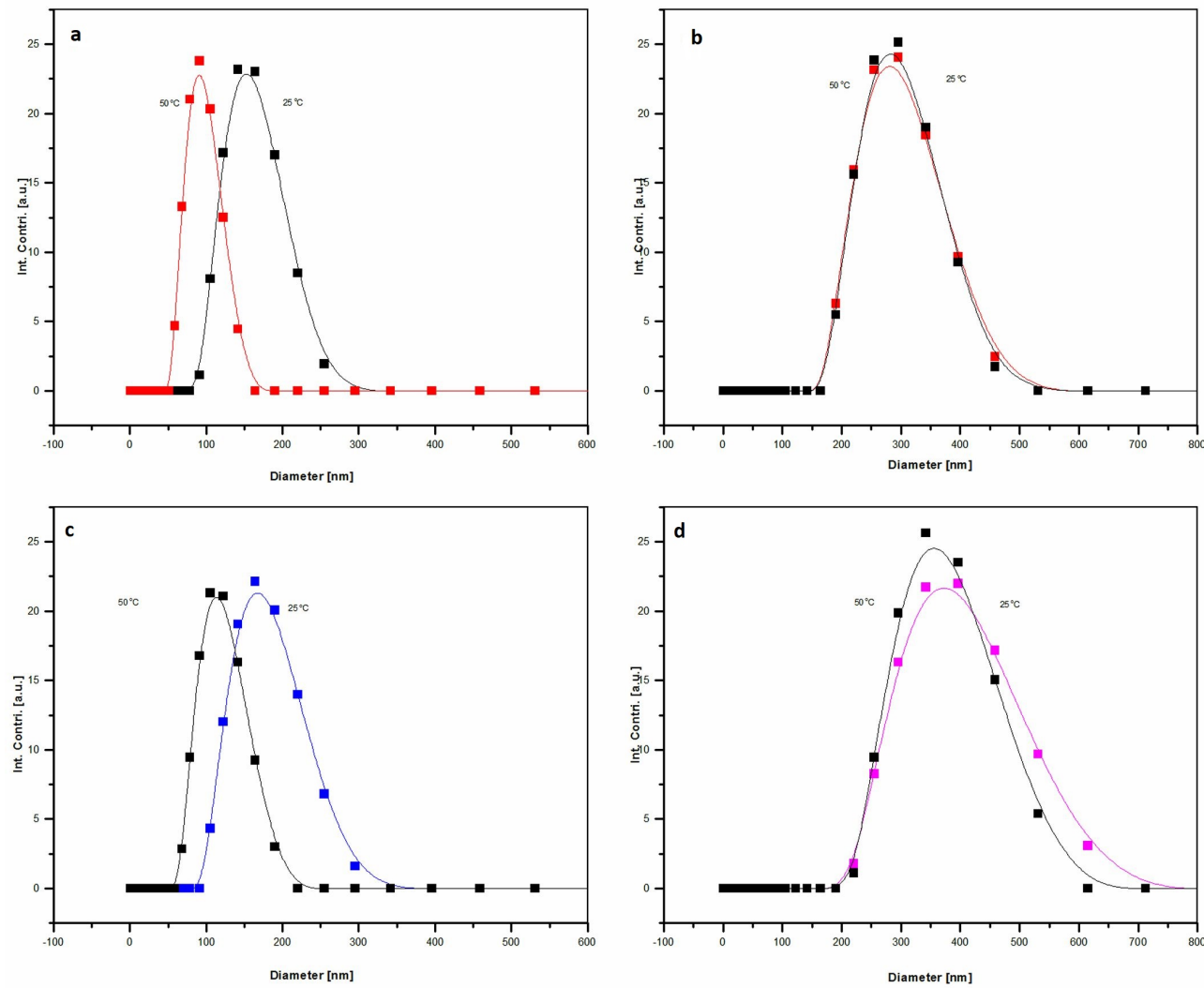

Fig. 4. Diameter of polymer microgel at various temperatures for (a) p(NIPAM-co-MAA)-1 at pH 2.78, (b) p(NIPAM-co-MAA)-1 at pH 8.36, (c) p(NIPAM-co-MAA)-2 at pH 2.78 and (d) p(NIPAM-co-MAA)-2 at $\mathrm{pH} 8.36$.

using $\mathrm{NaBH}_{4}$ as a reducing agent, by UV-Vis spectroscopy. The reduction in absorption peak intensity at $400 \mathrm{~nm}$ due to nitrophenolate ion $\left(\mathrm{NP}^{-}\right)$was plotted against time; the apparent rate constant $\mathrm{k}_{\mathrm{app}}$ was calculated from the slope of the linear curve.

\subsubsection{Catalysts synthesized at $\mathrm{pH} 2.78$}

The surface charge for pure copolymer $\mathrm{p}$ (NIPAM-co-MAA)-2 at any $\mathrm{pH}$ value is greater than that of $p$ (NIPAM-co-MAA)-1 microgel. This increase in charge density enhances the particle size due to which p(NIPAM-co-MAA)-2 can accommodate more metal particles in comparison to $\mathrm{p}$ (NIPAM-co-MAA)-1 and shows enhanced catalytic efficiency. The catalytic efficiency was examined at various stages of reaction by noting the reduction in the peak intensity at $400 \mathrm{~nm}$ caused by $\pi-\pi^{*}$ allowed transition in $\mathrm{NP}^{-}$versus reaction time, indicating the consumption of reactants.
The formation of 4-AP was confirmed by color change and the appearance of a peak with low intensity at $300 \mathrm{~nm}$ caused by the $n-\delta^{*}$ forbidden transition of amino groups. In the absence of catalyst or $\mathrm{NaBH}_{4}$ no reduction in the peak intensity was observed which clearly indicates that the reaction is kinetically restricted, although it is thermodynamically feasible. The UV-Vis spectra obtained for $\mathrm{p}$ (NIPAM-co-MAA)-2-Ag and $\mathrm{p}$ (NIPAM-co-MAA)-2-Au synthesized at $\mathrm{pH}$ 2.78 are shown in Fig. 5.

The comparative catalytic activity was evaluated from the apparent rate constant $\mathrm{k}_{\mathrm{app}}$ value, which was calculated from the slope of $\ln \mathrm{A}$ against time, where $A=A_{o} / A_{t},\left(A_{o}\right.$ is the initial absorbance and $A_{t}$ is the absorbance at any time $t$ of $4-\mathrm{NP}^{-}$). The $\mathrm{k}_{\text {app }}$ obtained for $\mathrm{p}$ (NIPAM-coMAA)-1-Ag was $0.16134 \mathrm{~min}^{-1}$ which is less than 

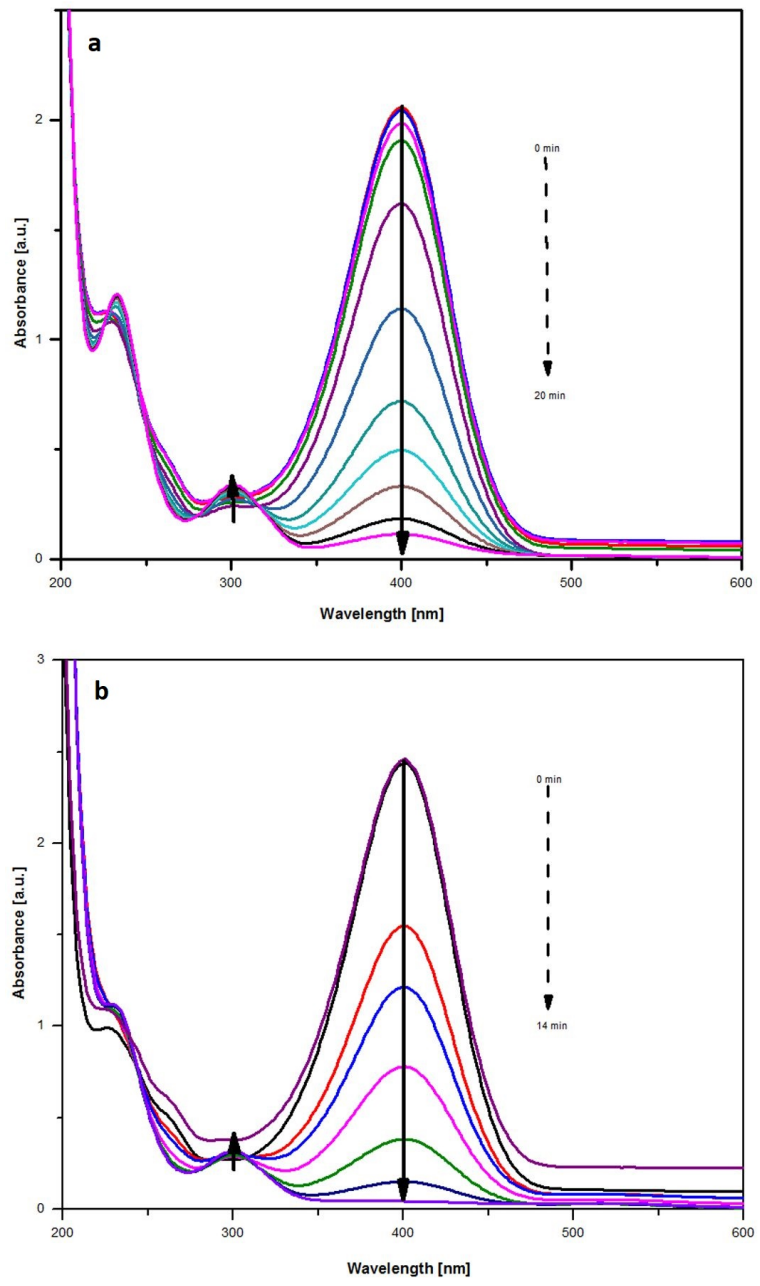

Fig. 5. UV-Vis spectra for the catalysts synthesized at pH 2.78 (a) p(NIPAM-co-MAA)-2-Ag and (b) p(NIPAM-co-MAA)-2-Au.

the $\mathrm{k}_{\mathrm{app}}$ value obtained for $\mathrm{p}$ (NIPAM-co-MAA)-2Ag, $0.20656 \mathrm{~min}^{-1}$ shown in Fig. 6. This increase in catalytic property is caused by the large size of microgel particles, which can be explained in two ways: first, the large size particles accommodate more metal particles, and second, the diffusion of reactants to the catalyst surface and removal of products from polymer network is facilitated. An induction time was observed for all prepared catalysts at $\mathrm{pH} 2.78$, which was due to the small size of microgel particles, restricting the penetration of reactants inside the polymer network and took some time to come in contact with the metal surface (Fig. 6a).
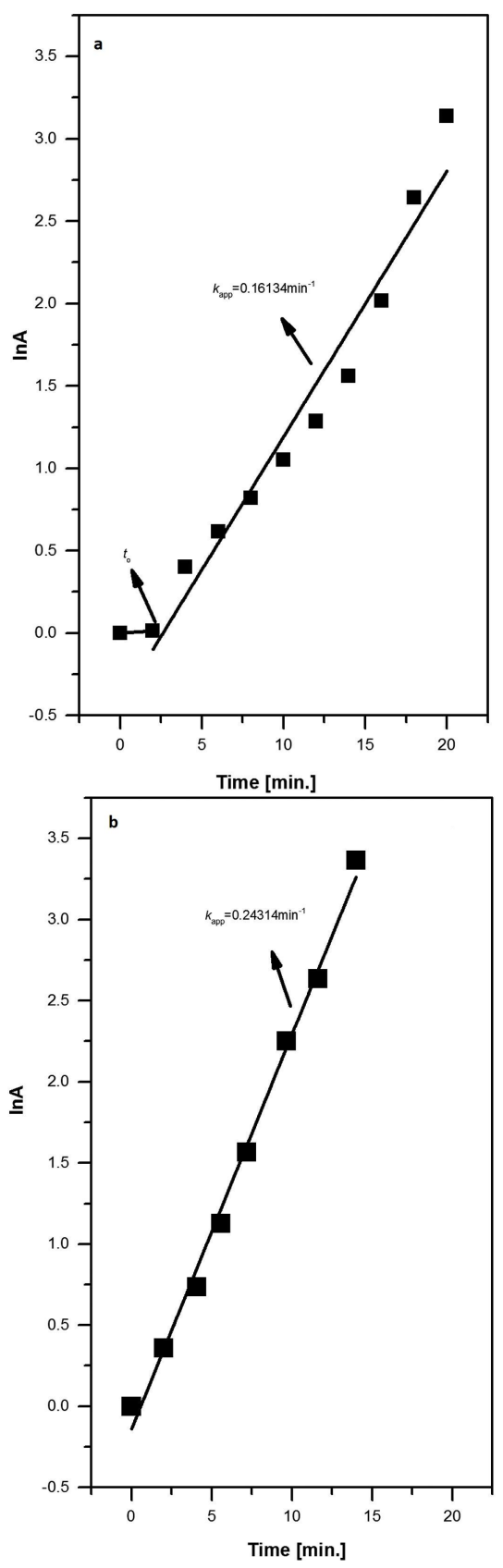

Fig. 6. Plot of $\ln A$ vs. time for the conversion of 4-NP by p(NIPAM-co-MAA)-1-Ag synthesized at (a) pH 2.78 and (b) pH 8.36.

The catalytic efficiency depends also on the type of metal nanoparticles entrapped in crosslinked polymer network. In this study, we have stabilized $\mathrm{Ag}$ and $\mathrm{Au}$ nanoparticles in cross-linked polymer network for comparative study. The size of $\mathrm{Au}$ nanoparticles was $3.5 \mathrm{~nm}( \pm 0.5 \mathrm{~nm})$ and 
Table 2. Obtained $\mathrm{k}_{\mathrm{app}}$ values for all catalysts synthesized at different $\mathrm{pH}$ values used for reduction process.

\begin{tabular}{ccc}
\hline Sample code & $\begin{array}{c}\text { Synthesis } \\
\mathrm{pH}\end{array}$ & $\begin{array}{c}\mathrm{k}_{\text {app }} \\
{\left[\mathrm{min}^{-1}\right]}\end{array}$ \\
\hline \hline p(NIPAM-co-MAA)-1-Ag & 2.78 & 0.16134 \\
& 8.36 & 0.24314 \\
p(NIPAM-co-MAA)-2-Ag & 2.78 & 0.20656 \\
& 8.36 & 0.30259 \\
p(NIPAM-co-MAA)-1-Au & 2.78 & 0.19634 \\
& 8.36 & 0.34112 \\
p(NIPAM-co-MAA)-2-Au & 2.78 & 0.23388 \\
& 8.36 & 0.5556 \\
\hline
\end{tabular}

that of $\mathrm{Ag}$ nanoparticles was $6.5 \mathrm{~nm}( \pm 0.5 \mathrm{~nm})$ as shown in Fig. 3. This small size makes Au based catalysts more efficient as compared to Ag particles containing catalysts, which is demonstrated by their greater $\mathrm{k}_{\mathrm{app}}$ values tabulated in Table 2 .

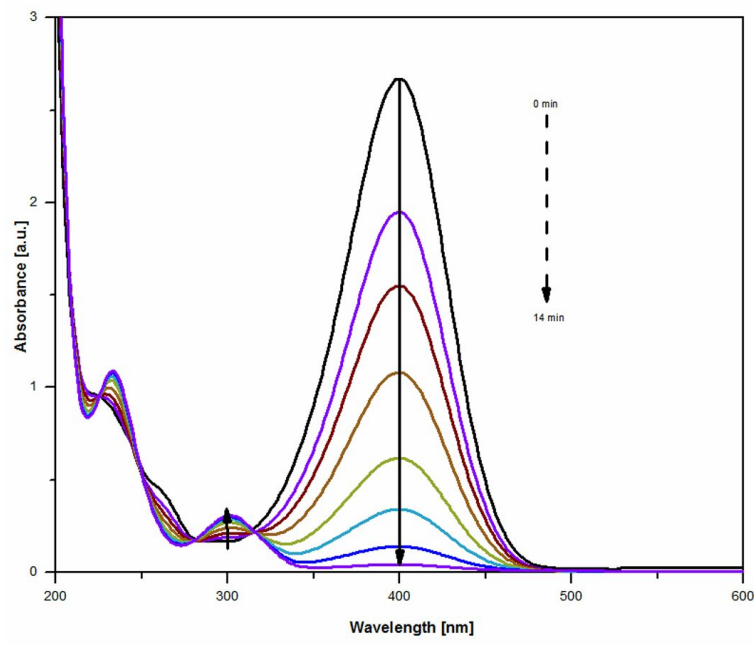

Fig. 7. UV-Vis spectra for $\mathrm{p}$ (NIPAM-co-MAA)-1-Ag synthesized at $\mathrm{pH} 8.36$.

\subsubsection{Catalysts synthesized at pH 8.36}

The charge density at $\mathrm{pH} 8.36$ is large due to ionization of carboxyl groups in polymer chains. The particles changed to swollen state, due to which they were able to provide more space and attraction for metal ions to come inside the polymer network. The UV-Vis spectra obtained for $\mathrm{p}$ (NIPAM-co-MAA)-1-Ag synthesized at $\mathrm{pH} 8.36$ are presented in Fig. 7. Again $\mathrm{k}_{\text {app }}$ for $\mathrm{p}$ (NIPAM-co-MAA)-1-Ag $\left(0.24314 \mathrm{~min}^{-1}\right)$ is less than the $\mathrm{k}_{\mathrm{app}}$ for $\mathrm{p}$ (NIPAM-co-MAA)-2-Ag $\left(0.30259 \mathrm{~min}^{-1}\right)$. The linear trend for $\mathrm{p}$ (NIPAMco-MAA)-1-Ag synthesized at $\mathrm{pH} 8.36$ is shown in Fig. 6b. No induction time was observed in this case, because the reactants take negligible time to come in contact with metal surface and quickly change into products due to the large size of the polymer network. The $\mathrm{k}_{\text {app }}$ values obtained for the catalysts synthesized at $\mathrm{pH} 8.36$ were found greater than those for the catalysts synthesized at $\mathrm{pH} 2.78$, which confirms the dependence of catalyst efficiency on the size of polymer microgel network. Due to the large size of polymer network, the reactant molecules easily penetrate inside it forming a colloid with the entrapped metal particles, and a chemical change occurs. After the formation of the products, the formed materials come out easily from the network.

At $\mathrm{pH}$ 8.36, $\mathrm{Au}$ based catalysts were found again more efficient than other $\mathrm{Ag}$ particles containing catalysts. The highest $\mathrm{k}_{\mathrm{app}}\left(0.5556 \mathrm{~min}^{-1}\right)$ was obtained for p(NIPAM-co-MAA)-2-Au, due to the large size of pure microgel particles and small size of entrapped Au NPs. The pure microgel particles leave enough space for Au particles, and small size of Au NPs provides a large surface area for the reactants to undergo a chemical change. The $k_{a p p}$ values for all catalysts synthesized at $\mathrm{pH} 2.78$ and 8.36 are given in Table 2 .

\section{Conclusions}

The responsive cross-linked polymer microgels $\mathrm{p}$ (NIPAM-co-MAA)s have been synthesized 
successfully by free radical emulsion polymerization with varying amount of MAA. Small size Ag and $\mathrm{Au}$ nanoparticles were successfully incorporated into the polymer network at $\mathrm{pH} 2.78$ and 8.36. The synthesized doped materials were used as catalysts for the reduction of 4-NP successfully. The results revealed that the catalysts synthesized at $\mathrm{pH} 8.36$ show enhanced catalytic activity compared to the catalysts prepared at $\mathrm{pH} 2.78$. Also it was confirmed that hybrid microgels composed of gold nanoparticles were more efficient than silver based hybrid microgels. Most importantly, it was proven in this study that $\mathrm{p}$ (NIPAM-co-MAA)s cross-linked polymer microgels can be used at different $\mathrm{pH}$ values for the immobilization of different metal nanoparticles and then can be applied as a catalyst for various organic reactions.

\section{Acknowledgements}

The Higher Education Commission of Pakistan is gratefully acknowledge for financial support under the Project No.: 21-718/ SRGP/R\&D/HEC/2016.

\section{References}

[1] Wanakule P., Liu G.W., Fleury A.T., Roy K., J. Control. Release, 162 (2012), 429.

[2] Moon H.J., Ko D.Y., PARK M.H., Joo M.K., JEOng B., Chem. Soc. Rev., 41 (2012), 4860.

[3] Han D.M., Zhang Q.M., Serpe M.J., Nanoscale, 7 (2015), 2784

[4] Marguet M., Bonduelle C., LecommanDOUX S., Chem. Soc. Rev., 42 (2013), 512.

[5] Zhang Q.M., Xu W., Serpe M.J., Angew. Chem. Int. Ed., 53 (2014), 4827.

[6] Herves P., Lorenzo M.P., Marzan L.M.L., DZUBiella J., Lu Y., Ballauff M., Chem. Soc. Rev., 41 (2012), 5577
[7] Chang A., Wu Q., Du X., Chen S., Sheng J., Song Q., XIE J., Wu W., Chem. Commun., 52 (2016), 4525 .

[8] Lu Y., Ballauff M., Prog. Polym. Sci., 36 (2011), 767.

[9] Yu Y.L., Zhang M.J., XIE R., Ju X.J., Wang J.Y., PI S.W., CHU L.Y., J. Colloid Interf. Sci., 376 (2012), 97.

[10] Luo Q., Liu P., Guan Y., Zhang Y., ACS Appl. Mater. Inter., 2 (2010), 760.

[11] SAhiner N., AtTA A.M., YASAR A.O., AlLohedan H.A., EzZAT A.O., Colloid. Surf. A, 482 (2015), 647.

[12] Zhang B., Sun B., Li X., Yu Y., Tian Y., Xu X., JIN Z., Int. J. Biol. Macromol., 79 (2015), 392.

[13] Ye T., Bai X., Jiang X., Wu Q., Chen S., Qu A., Huang J., Shen J., Wu W., Polym. Chem., 7 (2016), 2847.

[14] Xu W., Gao Y., Serpe M.J., J. Mater. Chem. C, 2 (2014), 3873.

[15] Wu Q., Cheng H., Chang A., BaI X., LU F. Wu W., Chem. Commun., 50 (2014), 14217.

[16] LiU Y.Y., LiU X.Y., YANG J.M., Lin D.L., Chen X., ZHA L.S., Colloid. Surf, A, 393 (2012), 105.

[17] Contin A., Biffis A., STerchele S., DormBACH K., SChIPMANn S., Pich A., J. Colloid. Interf. Sci., 414 (2014), 41.

[18] Gema M, Gonzalez M.L., Mendicuti F., TaraZONA M.P., VALIENTE M., Macromolecules, 47 (2014), 6028.

[19] S Ahiner N., Colloid. Surf. A, 433 (2013), 212.

[20] Shah L.A., Chen W., SiddiQ M., Hu J., Dong A., YANG D., Chinese J. Chem., 33 (2015), 467.

[21] Shah L.A., Haleem A., Sayed M., SiddiQ M., $J$. Environ. Chem. Eng., 4 (2016), 3492.

[22] Naeem H., Farooqi Z.H., Shah L.A., SiddiQ M., J. Polym. Res., 19 (2012), 1.

[23] Wu W., Zhou T., Berliner A., Banerjee P., Zhou S., Chem. Mater., 22 (2010), 1966.

Received 2017-04-03 Accepted 2017-08-25 